\title{
EL ASENTAMIENTO DE LA POBLACION ESPAÑOLA SOBRE EL TERRITORIO
}

\author{
$311.312(46)$ \\ por \\ Ignacio Ballester Ros
}

SUMARIO: I. INTRODUCCION.-II. TRASCENDENCIA DE LAS FORMAS DE ASENTAMIENTO DE LA POBLACION.-III. LAS ENTIDADES DE POBLACION Y SU DISTRIBUCION: 1. EN EL CONJUNTO Nacional. 2. Por Regiones. 3. Por Zonas comerciales.-IV. LA DISTRIBUCION DE LA POBLACION URBANA Y RURAL POR REGIONES Y AREAS COMERCIALES.-V. EL INDICE DE CONCENTRACION DE LA POBLACION ESPAÑOLA.

\section{INTRODUCCION}

Se acaba de publicar la undécima edición, correspondiente al año 1975, del Anuario del Mercado Español, que patrocina el Banco Español de Crédito. La mayor parte de sus trabajos tienen un enfoque preferentemente comercial; pero, al propio tiempo, tienen un marcado interés por su proyección provincial y municipal. Esta monografía, ya clásica, contiene, además, los resultados de investigaciones especialmente dirigidas al estudio de aspectos fundamentales de la Vida local.

En esta monografía se mantiene la misma estructura de las anteriores, así como su división en cinco partes: la primera, dedicada a la "Geografía del mercado", ofrece la cuota de mercado de las áreas comerciales, configuradas en el Atlas Comercial de España que editó el Consejo Superior de Cámaras de Comercio, Industria y Navegación; en la segunda, dedicada a las «Fuentes de riqueza e instrumentos de mercado", se presentan los consumos energéticos, los índices de capacidad de compra provincial y las licencias comerciales en vigencia; en la tercera se ofrecen los índices de riqueza activa de las áreas comerciales y los datos estadísticos sobre los 
Municipios con población superior a 3.000 habitantes, trabajo este último de periodicidad bienal; la cuarta comprende los indicadores del mercado, y la quinta los índices de vivacidad de las Provincias españolas y el análisis de los movimientos migratorios interiores. En trabajos anteriores, publicados en esta Revista, se ha expuesto esta sistemática con un mayor detalle.

Cada año se insertan en el expresado Anuario los resultados de algunas investigaciones especiales. En el presente se trata de dos trabajos de indudable interés: «El índice de concentración de la población de las áreas comerciales» $\mathrm{y}$ «La distribución provincial de la exportación española», trabajo este último que, iniciado en el año anterior, se trata en éste.'con mayor amplitud, y "constituye una de las informaciones más útiles para abordar los problemas del desarrollo regional», en boca de sus autores.

Desde la aparición del citado Anuario, esta Revista ha dedicado una crónica estadística anual al comentario de algunos de sus trabajos, escogidos entre los publicados, de forma tal que todos ellos han sido objeto de atención en algún momento.

Dada la riqueza informativa de este Anuario, que imposibilita la redacción de una crónica en la que se dé una visión global de su contenido con la suficiente profundidad de análisis, parece conveniente dedicar este trabajo al estudio de las formas de asentamiento de la población sobre el territorio, y del índice de concentración de la población en las áreas comerciales, que ha sido objeto de investigación especial por el equipo de estadísticos y economistas que elabora el Anuario.

Dos hechos avalan esta conveniencia: el carácter decenal de los datos básicos, como sólo permite su análisis cada diez años, y è interés en conocer la situación actual del asentamiento de la población sobre el territorio, después de transcurrido un decenio durante el que se intensificaron los movimientos migratorios interiores y se acentuó la despoblación en amplias áreas del territorio nacional.

\section{TRASCENDENCIA DE LAS FORMAS DE ASENTAMIENTO DE LA POBLACION}

Son ciertamente muy variadas las formas de asentamiento de la población sobre el territorio en las distintas áreas geográficas del país. Las posibilidades fisiográficas, los medios naturales de co- 
municación, la capacidad productiva del suelo, las características étnicas y sicológicas y los niveles culturales de los habitantes, las actividades económicas y profesionales que desarrollan, que suponen, en esencia, una modificación del medio por la acción humana y otros factores que condicionan el habitat, determinan las diferencias entre unas y otras Regiones. Existen también condicionantes históricos que han influido en algunas formas de asentamiento.

En el trabajo que comentamos se afirma muy acertadamente que si importante es "el conocimiento de la cifra de la población que agrupan las distintas Zonas, Areas o Provincias», igualmente lo es conocer «la forma en que los habitantes se distribuyen sobre la superficie de aquellos espacios para determinar, en función de la población, las posibilidades y características de su actividad mercantil». Es evidente que la dispersión o concentración de la población condiciona fuertemente las posibilidades de penetración comercial, y que de las características fisiográficas y humanas, que constituyen el habitat, pueden derivarse distintos niveles de renta y hábitos de compra.

Desde la perspectiva demográfica estricta, es igualmente importante el estudio del fenómeno del asentamiento de la población, que equivale a la localización geográfica de los poblados o «Entidades de Población", concepto puramente estadístico en el momento presente, y cuyo nacimiento, modificación o extinción sería conveniente se regulara, en un futuro próximo, mediante un trámite sumario jurídico-administrativo. Estas Entidades de Población están constituidas, en síntesis, por "agrupaciones de edificios y viviendas en forma de calles y plazas», que forman núcleos habitables. Tanto el pequeño poblado, en forma simple y primaria, como el casco de la gran ciudad son Entidades de Población; la diferencia estriba en el grado de disponibilidad y disfrute de los servicios públicos y comunitarios de unas y otras por sus respectivos habitantes, y en la distinta estructura urbana.

Los movimientos migratorios interiores, intensificados en el último decenio, han determinado una acusada disminución de habitantes, especialmente jóvenes, en las Entidades rurales, y un número ciertamente importante de ellas han quedado despobladas. Se han registrado movimientos de población desde las pequeñas Entidades rurales hacia las capitales de los respectivos Municipios y de unas y otros hacia los Municipios cabezas de Partido judicial, hacia las capitales de Provincia y su hinterland, y más intensamente, fuera 
de su Provincia, hacia alejadas zonas industriales. El fenómeno de la concentración de la población en las grandes urbes parece irreversible; pero se atisba la posibilidad, a plazo medio, de una más racional ordenación de los asentamientos de población sobre el territorio mediante el Municipio-comarca y otras formas institucionales contempladas en el Proyecto de Ley de Bases del Estatuto Local, que faciliten el montaje de servicios públicos y comunitarios, y un grado de comodidad adecuado que, junto a un cambio de la estructura agraria, ha de determinar forzosamente una notable disminución del número de pequeñas Entidades rurales.

No son solamente comerciales los efectos que se deducen de las estructuras de las Entidades, como forma habitual de asentamiento de la población. Los efectos de todo orden en el ámbito de la Administración estatal, local e institucional, por la acción de los administrados, son patentes y se acrecientan de día en día. La planificación del desarrollo económico y social, y la aplicación de las directrices de la política económica y social en todos los sectores de la actividad nacional tiene como soporte demográfico indispensable la estructura del asentamiento de la población sobre el territorio.

\section{LAS ENTIDADES DE POBLACION Y SU DISTRIBUCION}

Tomando como base los datos que, referentes a las 65.138 Entidades singulares de Población habitadas, figuran en el Nomenclátor de las Ciudades, Villas, Lugares, Aldeas y demás Entidades de la población de España, editado por el Instituto Nacional de Estadística, como información complementaria del Censo de Población de 1970, se presentan en el Anuario que comentamos, por áreas comerciales, las distribuciones de frecuencia de las Entidades y de sus respectivas cifras de habitantes, en diez grupos de niveles de población y, además, la clasificación de la población en rural, intermedia o mixta, y urbana, considerando a la primera como la residente en Entidades de hasta 2.000 habitantes; a la segunda, como la que habita en Entidades de 2.001 a 10.000, y como urbana la residente en Entidades de población superior a 10.000 habitantes, criterio seguido por el Instituto Nacional de Estadística, de acuerdo con las recomendaciones de la Conferencia de Estadísticos Europeos de las Naciones Unidas. 
Sobre dichos datos, hemos elaborado una redistribución por Regiones y Zonas comerciales y algunos resúmenes numéricos que facilitan el análisis geográfico.

\section{EN EL CONJUNTO NACIONAL}

La distribución, según los diez niveles de población aludidos, de las Entidades de Población y de sus cifras de habitantes, por grupos de Entidades, se detalla en esta forma:

\begin{tabular}{|c|c|c|c|c|}
\hline GRUPOS DE ENTIDADES & \multicolumn{2}{|c|}{ ENTIDADES } & \multicolumn{2}{|c|}{ HABITANTES } \\
\hline & \multicolumn{4}{|c|}{ Cifras absolutas } \\
\hline Hasta 100 habitantes $\ldots \ldots \ldots$ & 44.426 & \multirow{4}{*}{63.188} & 1.541 .100 & \multirow{4}{*}{8.859 .098} \\
\hline De $\begin{array}{llllllll}101 & \text { a } & 500 & \ldots & \ldots & \ldots & \ldots & \ldots\end{array}$ & 14.340 & & 3.088 .877 & \\
\hline 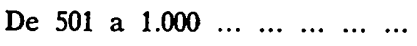 & 2.784 & & 1.945 .036 & \\
\hline De 1.001 a $2.000 \ldots \ldots$ & 1.638 & & 2.284 .085 & \\
\hline 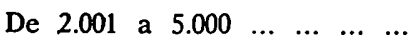 & 1.219 & \multirow{2}{*}{1.618} & 3.737 .679 & \multirow{2}{*}{6.540 .434} \\
\hline De 5.001 a $10.000 \ldots \ldots \ldots$ & 399 & & 2.802 .755 & \\
\hline De 10.001 a $20.000 \ldots \ldots \ldots$ & 174 & \multirow{4}{*}{332} & 2.453 .680 & \multirow{4}{*}{18.410 .743} \\
\hline De 20.001 a $50.000 \ldots \ldots \ldots$ & 94 & & 2.649 .284 & \\
\hline De 50.001 a $150.000 \ldots \ldots \ldots$ & 46 & & 3.937 .294 & \\
\hline \multirow[t]{2}{*}{ De más de $150.000 \ldots \ldots \ldots$} & 18 & & 9.370 .485 & \\
\hline & \multicolumn{4}{|c|}{ Porcentajes } \\
\hline Hasta 100 habitantes $\ldots \ldots \ldots$ & 68,2 & \multirow{4}{*}{97,0} & 4,6 & \multirow{4}{*}{25,2} \\
\hline De 101 a $500 \ldots \ldots \ldots \ldots$ & 22,0 & & 9,1 & \\
\hline De 501 a $1.000 \ldots \ldots \ldots$ & 4,3 & & 5,7 & \\
\hline De 1.001 a $2.000 \ldots \ldots \ldots \ldots$ & 2,5 & & 6,8 & \\
\hline De 2.001 a $5.000 \ldots \ldots \ldots \ldots$ & 1,9 & \multirow{2}{*}{2,5} & 11,1 & \multirow{2}{*}{19,3} \\
\hline De 5.001 a $10.000 \ldots \ldots \ldots$ & 0,6 & & 8,2 & \\
\hline De 10.001 a $20.000 \ldots \ldots \ldots$ & 0,3 & \multirow{4}{*}{0,5} & 7,3 & \multirow{4}{*}{54,5} \\
\hline De 20.001 a $50.000 \ldots \ldots \ldots$ & 0,2 & & 7,8 & \\
\hline De 50.001 a $150.000 \ldots \ldots \ldots$ & - & & 11,7 & \\
\hline De más de $150.000 \ldots \ldots$ & - & & 27,7 & \\
\hline
\end{tabular}

El desarrollo, por niveles de población, del número de Entidades de Población se presenta en razón inversa al de sus respectivas cifras de habitantes. A pesar de la disminución de población o del despoblamiento de Entidades, las pequeñas Entidades son todavía muy numerosas. Más de las dos terceras partes de las Entidades no rebasan los cien habitantes. En cuatro de cada cinco Entidades residen a lo sumo quinientas personas. Sólo tres de cada cien Enti- 
dades tienen más de 2.000 habitantes. Esta distribución corresponde todavía a una economía predominantemente agraria.

Por lo que a la distribución de habitantes se refiere, es de señalar, a la vista de las cifras anteriores, que casi nueve millones de habitantes, la cuarta parte de la población, reside en Municipios de hasta 2.000 habitantes, lo que equivale a decir que de cada cuatro familias, una es todavia rural. La quinta parte de la población habita en Entidades de carácter mixto rural-urbano. En las Entidades más populosas, superiores a los 10.000 habitantes, viven más de la mitad de los españoles, por lo que se advierte una fuerte concentración de la población, ya que, en tan sólo 332 Entidades residen 18 millones y medio de habitantes. Esta concentración se manifiesta más claramente si consideramos que en las 64 Entidades mayores de 50.000 habitantes se ubican más de 13 millones de habitantes, es decir, que en ellas viven 39 de cada cien españoles.

En resumen, pudiera decirse que, junto a una ruralidad demográfica que se debilita acentuadamente, se pone de manifiesto una inusitada concentración de los habitantes en un número reducido de Entidades populosas.

\section{POR REGIONES}

Contemplada la distribución de Entidades y de sus poblaciones en el conjunto nacional, vamos a examinarla por Regiones. Consideramos las nueve siguientes que, dada la naturaleza del fenómeno, se adaptan mejor a su estudio y localización geográficos.

\begin{tabular}{|c|c|c|c|c|}
\hline Regiones & $\begin{array}{l}\text { Núm. de } \\
\text { Entidades }\end{array}$ & $\begin{array}{l}\text { Porcen- } \\
\text { tajes }\end{array}$ & $\begin{array}{c}\text { Núm. de } \\
\text { Municipios }\end{array}$ & $\begin{array}{l}\text { Entidades } \\
\text { por Munic. }\end{array}$ \\
\hline Galicia y Asturias & 39.411 & 60,5 & 390 & 101,1 \\
\hline Orla cantábrica (resto) $\ldots \ldots \ldots$ & 2.261 & 3,5 & 280 & 8,1 \\
\hline Submeseta Norte $\ldots \ldots \ldots \ldots$ & 6.079 & 9,3 & 2.572 & 2,4 \\
\hline Submeseta Sur $\ldots \ldots \ldots \ldots \ldots$ & 2.705 & 4,2 & 1.568 & 1,7 \\
\hline $\begin{array}{lllllllll}\text { Andalucía } & \ldots & \ldots & \ldots & \ldots & \ldots & \ldots & \ldots & \ldots\end{array}$ & 3.741 & 5,7 & 796 & 4,7 \\
\hline $\begin{array}{lllllll}\text { Valle del } & \text { Ebro } & \ldots & \ldots & \ldots & \ldots & \ldots\end{array}$ & 4.366 & 6,7 & 1.586 & 2,8 \\
\hline Litoral catalán-levantino ... ... ... & 5.507 & 8.5 & 1.374 & 4,0 \\
\hline $\begin{array}{llllllllll}\text { Canarias } & \ldots & \ldots & \ldots & \ldots & \ldots & \ldots & \ldots & \ldots\end{array}$ & 1.068 & 1,6 & 87 & 12,3 \\
\hline
\end{tabular}

De las cifras anteriores se deduce, en primer término, el gran número de Entidades de Población existentes en Galicia y Asturias, seis de cada diez. Los habitantes del resto del país se ubican en solamente 25.727 Entidades. Cada una de las siguientes Regiones: Submeseta Norte, Litoral catalán-levantino, Valle del Ebro y Anda- 
lucía, por este orden, tienen más del 5 por 100 de Entidades; las demás Regiones tienen un menor número relativo de Entidades.

Para mejor conocer la densidad de la distribución de Entidades por Regiones hemos tenido en cuenta el número de Municipios de cada una de ellas y hemos hallado el promedio de Entidades por Municipio, para compararlo con el promedio nacional (65.138 Entidades entre 8.653 Municipios) de 7,5.

Fácilmente se advierte la concentración de Entidades en Galicia $y$ Asturias, con más de cien Entidades por Municipio, concentración que, en realidad, expresa una acusada dispersión de los habitantes sobre el área territorial. Sólo el resto de la Orla cantábrica y Canarias ofrecen un promedio superior al nacional. En la mayor parte del país el promedio indicado es inferior al nacional, lo que, en principio, indica una mayor concentración de la población en un número relativamente reducido de Entidades.

En general, las Zonas comerciales ( 52 en el conjunto del país) siguen la ‘ónica de la Región a que pertenecen; son de señalar, sin embargo, cuatro excepciones: Vitoria y León, con nueve y siete Entidades respectivamente, por encima del promedio regional, y Guipúzcoa, con cuatro, por debajo de él. Es de destacar a Murcia, con treinta y dos Entidades por Municipio.

En resumen, se hace patente la fuerte concentración de Entidades en el Noroeste, Canarias y Murcia, que contrasta con un número relativamente pequeño en el resto del país.

\section{Por Zonas comerciales}

Operando con una mayor desagregación, analizaremos la distribución de las Entidades de Población por Regiones y Zonas comerciales, según el carácter rural, mixto o urbano de sus habitantes. Para ello, sobre la información contenida en la monografía comen. tada, hemos elaborado esta tabla:

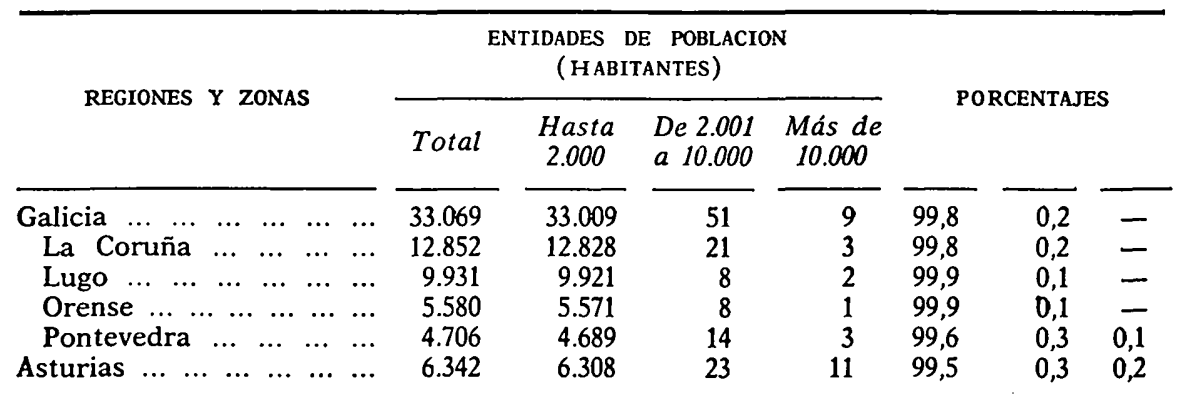




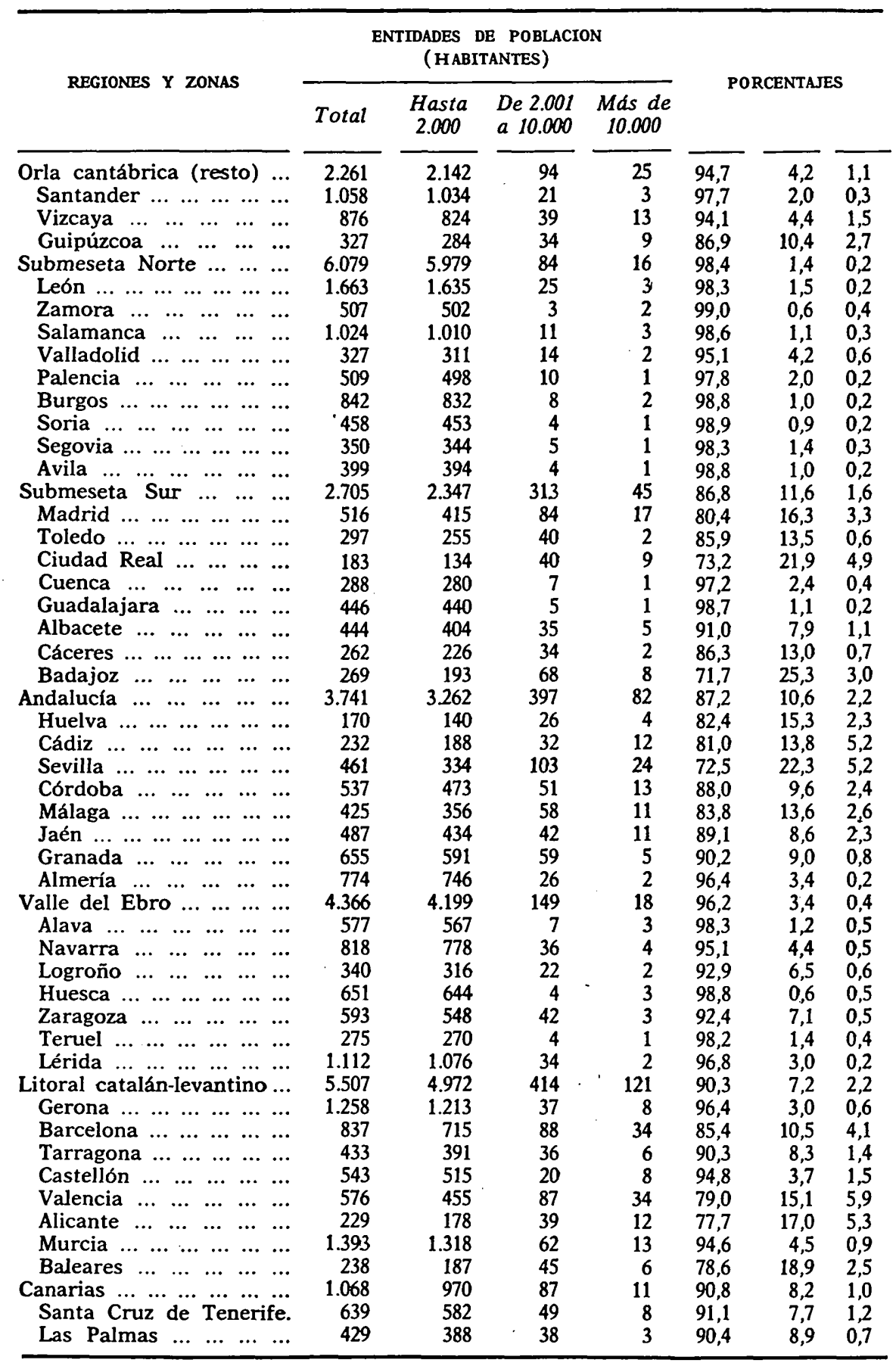


Las Entidades rurales se sitúan preferentemente en las Regiones de Galicia, Asturias y la Submeseta Norte, y aun con valores inferiores al promedio nacional, en el Valle del Ebro, el Litoral catalán-levantino y Canarias. Su proporción es menor en las restantes Regiones. Consecuentemente, destacan las Zonas comerciales de Galicia y Asturias, con más del 99 por 100, con Santander a menor nivel. En la Submeseta Norte, a excepción de Valladolid, todas las Zonas superan el 98 por 100 de Entidades rurales, y a ellas pueden asimilarse Guadalajara y Cuenca. En el Valle del Ebro superan el promedio nacional Alava, Huesca y Teruel. En el Litoral catalánlevantino, junto a Zonas por encima del 94, aparecen valores inferiores al 80 por 100. En la Submeseta Sur y Andalucía aparecen los mínimos nacionales: Ciudad Real, Sevilla y Badajoz, con sólo algo más del 70 por 100 de Entidades rurales, que contrastan con las tasas más altas de Almería, Granada y Albacete. En resumen, las Entidades rurales cubren, de manera predominante, una amplia área territorial, sin solución de continuidad, que comprende el Noroeste, el Valle del Duero y parte de la Orla cantábrica, y se prolonga por Alava, Guadalajara, Cuenca y Teruel, con Huesca aislada en los Pirineos.

Las Entidades urbanas predominan, por el contrario, en Andalucía, el Litoral catalán-levantino y, a mayor distancia, en la Submeseta Sur, Canarias y la Orla cantábrica. Destacan, en consecuencia, las Zonas de Andalucía, salvo Almería, con sus valores máximos en Cádiz y Sevilla; las del Litoral catalán-levantino, con los máximos nacionales de Valencia y Alicante, que superan a Cádiz y Sevilla, y descendiendo escalonadamente en valores las restantes; las de la Submeseta Sur, con la excepción de Cuenca y Guadalajara; las de Canarias, al igual que Guipúzcoa y Vizcaya en la Orla cantábrica. En las restantes Regiones destacan por encima del promedio nacional las Zonas de Valladolid, Alava y Navarra, Logroño, Huesca y Zaragoza, con valores similares o ligeramente superiores. En resumen, estas Entidades urbanas cubren, en forma predominante, una amplia área territorial, que comprende básicamente el centro, sur y este de la Península y parte del Valle del Ebro.

Las Entidades de carácter mixto urbano-rural se distribuyen sobre el territorio en forma extensa e irregular. Predominan en la Submeseta Sur, Andalucía, Canarias, Litoral catalán-levantino y Valle del Ebro, conformando una área que coincide en su mayor parte con la de predominio de las Entidades urbanas. Los valores 
entre las Zonas de una misma Región presentan frecuentemente acusadas diferencias. Las Zonas de la Submeseta Sur, a excepción de Cuenca y Guadalajara, de Andalucía, Canarias y el Litoral catalánlevantino, superan el promedio nacional, si bien destaca con el valor máximo nacional Badajoz, seguida de Sevilla y Ciudad Real. En el Valle del Ebro, sólo Zaragoza, Logroño, Navarra y Lérida superan moderadamente el promedio nacional, y de manera análoga se producen Guipúzcoa, Vizcaya y Valladolid.

\section{LA DISTRIBUCION DE LA POBLACION URBANA Y RURAL POR REGIONES Y AREAS COMERCIALES}

La información básica la tomamos del Anuario (páginas 16 a 19) y se detalla en la siguiente tabla, en la que se han hallado los totales y valores medios por Regiones.

\begin{tabular}{|c|c|c|c|c|c|c|}
\hline \multirow{2}{*}{ REGIONES Y AREAS } & \multicolumn{3}{|c|}{$\begin{array}{l}\text { NUMERO DE HABITANTES OUE } \\
\text { CONSTITUYEN LA POBLACION }\end{array}$} & \multicolumn{3}{|c|}{ PORCENTAJES } \\
\hline & Rural & Mixta & Urbana & & & \\
\hline 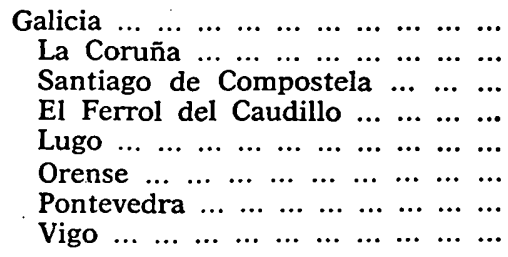 & $\begin{array}{r}1.818 .721 \\
272.712 \\
299.340 \\
102.336 \\
323.374 \\
358.468 \\
213.590 \\
248.901\end{array}$ & $\begin{array}{r}177.673 \\
28.691 \\
25.775 \\
16.138 \\
26.004 \\
27.958 \\
17.125 \\
35.982\end{array}$ & $\begin{array}{r}595.096 \\
184.372 \\
51.620 \\
75.464 \\
67.506 \\
63.542 \\
38.066 \\
114.526\end{array}$ & $\begin{array}{l}70,18 \\
56,14 \\
79,46 \\
52,77 \\
77,57 \\
79,67 \\
79,47 \\
62,32\end{array}$ & $\begin{array}{l}6,86 \\
5,91 \\
6,84 \\
8,32 \\
6,24 \\
6,21 \\
6,37 \\
9,01\end{array}$ & $\begin{array}{l}22,96 \\
37,95 \\
13,70 \\
38,91 \\
16,19 \\
14,12 \\
14,16 \\
28,67\end{array}$ \\
\hline 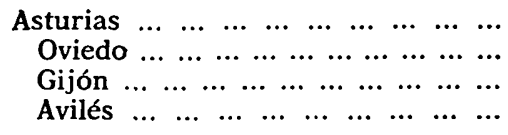 & $\begin{array}{r}490.039 \\
370.400 \\
61.979 \\
57.660\end{array}$ & $\begin{array}{r}113.874 \\
91.518 \\
11.849 \\
10.507\end{array}$ & $\begin{array}{r}401.259 \\
174.267 \\
159.806 \\
67.186\end{array}$ & $\begin{array}{l}48,75 \\
58,22 \\
26,53 \\
42,60\end{array}$ & $\begin{array}{r}11,33 \\
14,39 \\
5,07 \\
7,76\end{array}$ & $\begin{array}{l}39,92 \\
27,39 \\
68,40 \\
49,64\end{array}$ \\
\hline 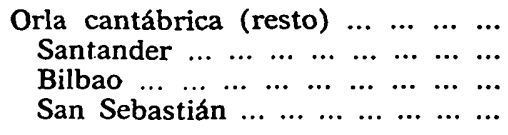 & $\begin{array}{l}479.236 \\
211.084 \\
164.692 \\
103.460\end{array}$ & $\begin{array}{r}439.433 \\
95.694 \\
184.559 \\
159.180\end{array}$ & $\begin{array}{r}1.255 .002 \\
160.815 \\
779.803 \\
314.384\end{array}$ & $\begin{array}{l}22,05 \\
45,14 \\
14,59 \\
17,93\end{array}$ & & $\begin{array}{l}57,73 \\
34,39 \\
69,07 \\
54,48\end{array}$ \\
\hline 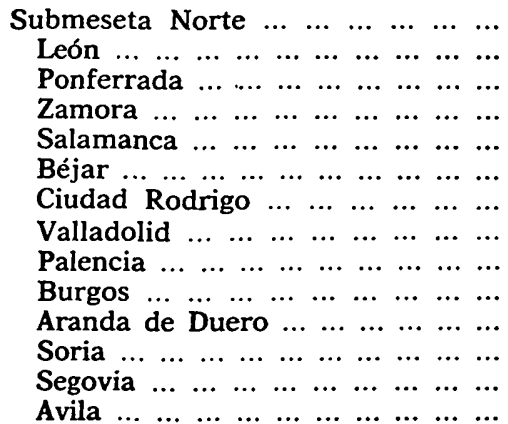 & $\begin{array}{r}1.345 .443 \\
236.317 \\
109.986 \\
166.865 \\
165.431 \\
18.673 \\
37.625 \\
114.503 \\
103.340 \\
94.330 \\
42.135 \\
57.904 \\
90.392 \\
107.942\end{array}$ & $\begin{array}{r}289.606 \\
52.497 \\
39.421 \\
13.724 \\
30.093 \\
- \\
3.090 \\
47.739 \\
39.295 \\
17.812 \\
2.822 \\
12.166 \\
17.514 \\
13.433\end{array}$ & $\begin{array}{r}884.848 \\
111.496 \\
22.838 \\
60.570 \\
125.132 \\
16.804 \\
11.694 \\
243.856 \\
58.327 \\
118.366 \\
18.183 \\
24.744 \\
41.880 \\
30.958\end{array}$ & $\begin{array}{l}53,39 \\
59,03 \\
63,85 \\
69,19 \\
51,59 \\
52,63 \\
71,79 \\
28,20 \\
51,42 \\
40,92 \\
66,73 \\
61,07 \\
60,35 \\
70,86\end{array}$ & $\begin{array}{r}11,49 \\
13,11 \\
22,89 \\
5,69 \\
9,38 \\
- \\
5,90 \\
11,76 \\
19,55 \\
7,73 \\
4,47 \\
12,83 \\
11,69 \\
8,82\end{array}$ & $\begin{array}{l}35,12 \\
27,85 \\
13,26 \\
25,12 \\
39,02 \\
47,37 \\
22,31 \\
60,05 \\
29,02 \\
51,35 \\
28,80 \\
26,10 \\
27,96 \\
20,32\end{array}$ \\
\hline
\end{tabular}


ESTADISTICA

\begin{tabular}{|c|c|c|c|c|c|c|}
\hline \multirow[t]{2}{*}{ REGIONES $Y$ AREAS } & \multicolumn{3}{|c|}{$\begin{array}{l}\text { NUMERO DE HABITANTES QUE } \\
\text { CONSTITUYEN LA POBLACION }\end{array}$} & \multicolumn{3}{|c|}{ PORCENTAJES } \\
\hline & Rural & Mixta & Urbana & & & \\
\hline 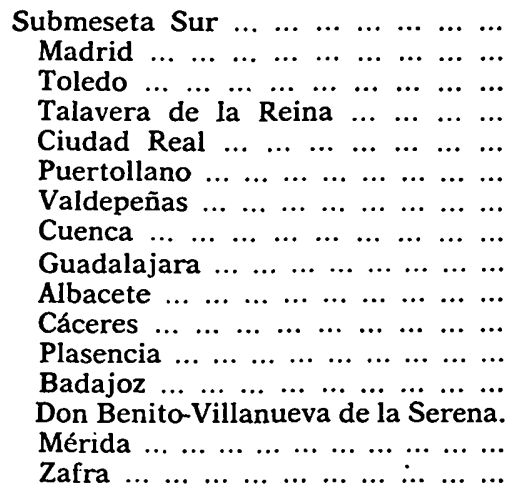 & $\begin{array}{r}982.637 \\
196.035 \\
39.151 \\
118.985 \\
44.774 \\
18.849 \\
12.971 \\
80.514 \\
81.760 \\
125.382 \\
81.004 \\
64.364 \\
42.372 \\
31.451 \\
24.120 \\
20.905\end{array}$ & $\begin{array}{r}1.240 .391 \\
345.326 \\
47.772 \\
85.554 \\
119.695 \\
13.988 \\
51.849 \\
18.409 \\
17.139 \\
118.233 \\
112.001 \\
33.264 \\
94.212 \\
90.940 \\
27.381 \\
64.628\end{array}$ & $\begin{array}{r}4.363 .504 \\
3.564 .087 \\
43.905 \\
39.889 \\
135.623 \\
50.609 \\
24.018 \\
33.980 \\
30.924 \\
146.664 \\
53.108 \\
26.897 \\
114.653 \\
38.038 \\
36.916 \\
24.193\end{array}$ & $\begin{array}{r}14,92 \\
4,77 \\
29,93 \\
48,68 \\
14,92 \\
22,59 \\
14,60 \\
60,58 \\
62,98 \\
32,13 \\
32,91 \\
51,69 \\
16,87 \\
19,60 \\
27,28 \\
19,05\end{array}$ & $\begin{array}{r}18,83 \\
8,41 \\
36,52 \\
35,00 \\
39,89 \\
16,76 \\
58,36 \\
13,85 \\
13,20 \\
30,29 \\
45,51 \\
26,71 \\
37,50 \\
56,69 \\
30,97 \\
58,90\end{array}$ & $\begin{array}{l}66,25 \\
86,81 \\
33,56 \\
16,32 \\
45,19 \\
60,65 \\
27,04 \\
25,57 \\
23,82 \\
37,58 \\
21,58 \\
21,60 \\
45,64 \\
23,71 \\
41,75 \\
22,05\end{array}$ \\
\hline 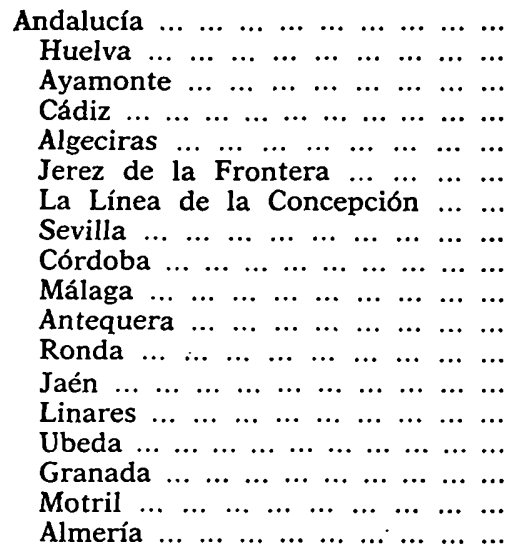 & $\begin{array}{r}32.28 \\
6.15 \\
30.84 \\
21.54 \\
38.05 \\
10.39 \\
134.11 \\
120.95 \\
102.940 \\
22.73 \\
42.86 \\
54.00 \\
25.66 \\
64.07 \\
253.18 \\
20.89 \\
150.67\end{array}$ & $\begin{array}{r}.649 .464 \\
105.574 \\
9.897 \\
40.285 \\
30.582 \\
52.935 \\
9.505 \\
448.634 \\
225.057 \\
142.316 \\
42.518 \\
34.768 \\
86.404 \\
23.498 \\
73.421 \\
212.362 \\
23.888 \\
87.820\end{array}$ & $\begin{array}{r}.208 .720 \\
130.036 \\
- \\
305.926 \\
74.754 \\
158.111 \\
51.021 \\
934.108 \\
402.737 \\
442.548 \\
46.599 \\
35.260 \\
148.737 \\
71.675 \\
40.279 \\
226.949 \\
25.121 \\
114.859\end{array}$ & $\begin{array}{r}12,05 \\
38,33 \\
8,18 \\
16,98 \\
15,28 \\
14,65 \\
8,84 \\
16,15 \\
14,97 \\
20,33 \\
37,97 \\
18,68 \\
21,24 \\
36,04 \\
36,56 \\
29,89 \\
42,64\end{array}$ & & $\begin{array}{l}48,54 \\
-81,14 \\
58,92\end{array}$ \\
\hline 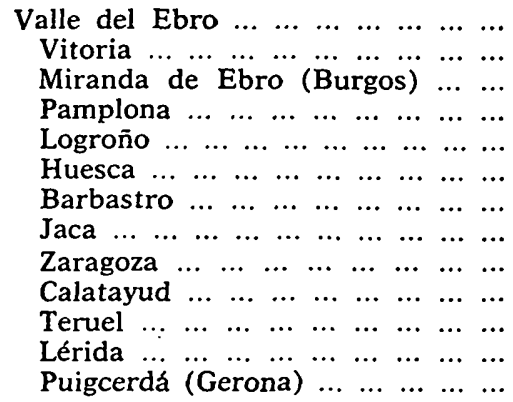 & $\begin{array}{r}804.769 \\
45.081 \\
11.091 \\
117.985 \\
91.203 \\
27.225 \\
34.925 \\
13.191 \\
155.969 \\
45.139 \\
68.728 \\
186.010 \\
7.222\end{array}$ & $\begin{array}{r}544.049 \\
28.573 \\
2.047 \\
123.042 \\
80.449 \\
9.667 \\
2.437 \\
9.936 \\
137.670 \\
6.682 \\
11.595 \\
127.533 \\
4.418\end{array}$ & $\begin{array}{r}144.640 \\
29.355 \\
187.327 \\
99.492 \\
33.076 \\
27.332 \\
- \\
460.386 \\
16.524 \\
20.614 \\
84.824 \\
-\end{array}$ & $\begin{array}{l}20,65 \\
26,10 \\
27,54 \\
33,64 \\
38,91 \\
53,98 \\
57,04 \\
20,68 \\
66,05 \\
68,40 \\
46,69 \\
62,04\end{array}$ & $\begin{array}{r}22,18 \\
13,09 \\
4,82 \\
28,72 \\
29,67 \\
13,82 \\
3,77 \\
42,96 \\
18,26 \\
9,78 \\
11,37 \\
32,01 \\
37,96\end{array}$ & $\begin{array}{c}43,73 \\
36,69 \\
47,27 \\
42,25 \\
- \\
61,06 \\
24,18 \\
20,22 \\
21,29 \\
-\end{array}$ \\
\hline 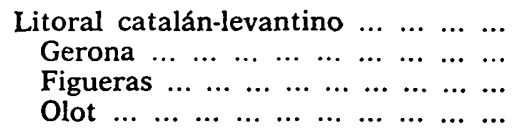 & $\begin{array}{r}1.423 .827 \\
102.699 \\
37.969 \\
24.478\end{array}$ & $\begin{array}{r}786.423 \\
89.991 \\
9.788 \\
-\end{array}$ & $\begin{array}{r}103.655 \\
75.332 \\
22.087 \\
18.062\end{array}$ & $\begin{array}{l}15,29 \\
38,32 \\
54,36 \\
57,54\end{array}$ & $\begin{array}{l}19,18 \\
33,58 \\
14,01 \\
-\end{array}$ & $\begin{array}{l}65,53 \\
28,11 \\
31,62 \\
42,46\end{array}$ \\
\hline
\end{tabular}




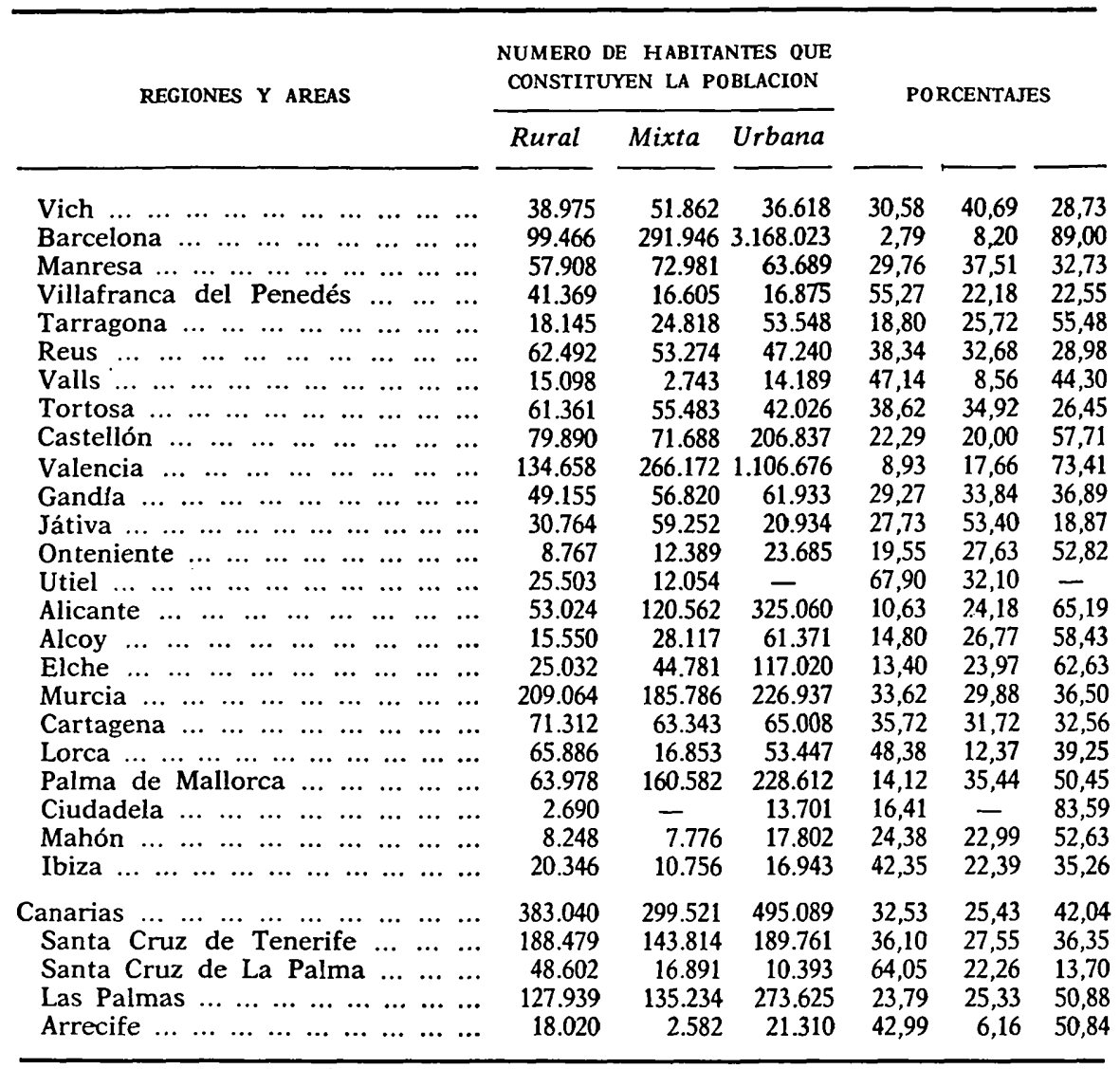

La población rural se sitúa preferentemente en las Regiones de Galicia, Asturias, Submeseta Norte, Valle del Ebro y Canarias, únicas cuyos porcentajes superan el promedio nacional, conformando una gran área que coincide fundamentalmente con la configurada en relación con las Entidades rurales; pero que la completa y amplía. La Orla cantábrica se aproxima al promedio nacional y las restantes Regiones ofrecen valores más modestos y similares entre sí. En todas las áreas de estas cinco Regiones predomina la población rural, salvo en Zaragoza y Las Palmas. Da idea, sin embargo, de la generalización del predominio de esta población el hecho de que en las demás Regiones aparecen hasta 31 áreas con valores superiores al promedio nacional. En resumen, de manera similar a la conclusión formulada con respecto a las Entidades rurales, la población rural cubre predominantemente una amplia área territorial 
que comprende el Noroeste, los Valles del Duero y Ebro, Canarias $y$ varias áreas del centro, sur y este de la Península.

La población urbana predomina, por el contrario, sobre una amplia área en el centro, sur y este de la Peninsula, similar en lo esencial con la descrita en relación con las Entidades urbanas; pero las áreas urbanas que comprenden son reducidas en número; así, en la Submeseta Sur sólo destaca Madrid, con una alta tasa, la segunda del país, que condiciona el porcentaje de la Región, y Puertollano; en Andalucía, seis; en el Litoral catalán-levantino, Barcelona, con la tasa más elevada del país, y otras siete, entre ellas Valencia y Alicante, y Ciudadela, la tercera área urbana de España; dos más en la Orla cantábrica y cuatro aisladas en otras Regiones.

La población de carácter mixto urbano-rural configura, en cuanto a las Regiones se refiere, un área territorial más restringida, ya que solamente comprende la Orla cantábrica, el Valle del Ebro, Andalucía y Canarias, si bien el Litoral catalán-levantino tiene un promedio sólo en una décima inferior al nacional. En cuanto a las áreas se refiere, destacan San Sebastián y Santander en el norte; Pamplona y Logroño, Jaca, Lérida y Puigcerdá en el Valle del Ebro; todas las andaluzas, a excepción de Cádiz y La Línea, y todas las canarias menos Arrecife. Sin embargo, destacan igualmente todas las áreas de la Submeseta Sur, a excepción de Madrid y Puertollano, Cuenca y Guadalajara, y las del Litoral catalán-levantino, salvo cinco, y otras dos en el Valle del Duero. En realidad, pues, se confirma el mayor peso del centro, sur y este, coincidiendo, al igual que ocurría con las Entidades, con el área territorial de predominio de la población urbana, aunque en forma mucho más amplia en cuanto al número de áreas afectadas se refiere.

\section{EL INDICE DE CONCENTRACION DE LA POBLACION ESPAÑOLA}

Teniendo en cuenta la población de las Entidades ubicadas en cada una de las 101 Areas comerciales, y aplicando el Indice de concentración de Gini, tan empleado para medir concentración de rentas, y que se estima igualmente aplicable a la concentración de la población, se han obtenido los índices de cada área comercial.

Los índices obtenidos varían desde un mínimo de 0,2359 , de Lugo, al máximo de 0,8764, de Ciudadela. En función de ellos, las áreas pueden agruparse en esta forma: 


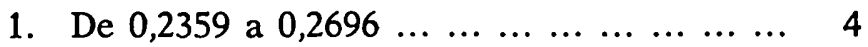

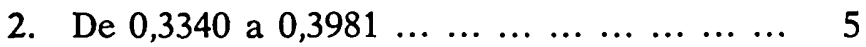

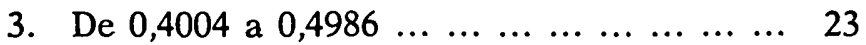

4. De 0,5050 a $0,5980 \ldots \ldots \ldots \ldots \ldots \ldots$

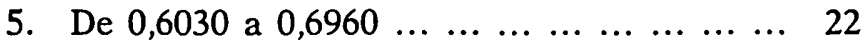

6. De 0,7011 a $0,7923 \ldots \ldots \ldots \ldots \ldots \ldots \ldots$

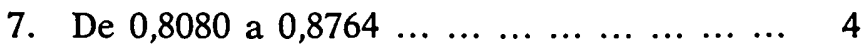

Igualmente se distribuyen por Regiones y grupos de índices, así:

\begin{tabular}{|c|c|c|c|c|c|c|c|c|}
\hline \multirow{2}{*}{ REGIONES } & \multicolumn{7}{|c|}{ GRUPOS DE INDICES } & \multirow{2}{*}{ Total } \\
\hline & 1 & 2 & 3 & 4 & 5 & 6 & 7 & \\
\hline $\begin{array}{llllllll}\text { Galicia } & \ldots & \ldots & \ldots & \ldots & \ldots & \ldots & \ldots\end{array}$ & 4 & - & 2 & 1 & - & - & - & 7 \\
\hline $\begin{array}{cccccc}\text { Asturias } & \ldots & \ldots & \ldots & \ldots & \ldots\end{array} \ldots$ & - & - & 1 & - & 1 & 1 & - & 3 \\
\hline Orla cantábrica (resto) ........ & - & - & - & 1 & 2 & - & - & 3 \\
\hline Submeseta Norte ... ... ... ... & - & 4 & 5 & 2 & 2 & - & - & 13 \\
\hline Submeseta Sur $\ldots \ldots \ldots$ & - & - & 5 & 7 & 1 & 1 & 1 & 15 \\
\hline $\begin{array}{lllllll}\text { Andalucía } & \ldots & \ldots & \ldots & \ldots & \ldots & \ldots\end{array}$ & - & - & 1 & 5 & 6 & 4 & 1 & 17 \\
\hline Valle del Ebro $\ldots \ldots \ldots \ldots$ & - & - & 3 & 5 & 1 & 3 & - & 12 \\
\hline Litoral catalán-levantino .... & - & - & 5 & 9 & 7 & 4 & 2 & 27 \\
\hline Canarias $\ldots \ldots \ldots \ldots \ldots$ & - & 1 & 1 & - & 2 & - & - & 4 \\
\hline Total $\ldots \ldots \ldots \ldots$ & 4 & 5 & 23 & 30 & 22 & 13 & 4 & 101 \\
\hline
\end{tabular}

Los índices confirman las conclusiones fijadas en los apartados anteriores. Los máximos corresponden a Barcelona, Madrid, Ciudadela y La Línea; y los inmediatos inferiores se asignan a Puertollano, antes destacado junto con Madrid; a Valencia, Tarragona, Alcoy y Mahón, Algeciras, Málaga y Linares, y a Zaragoza, Vitoria y Miranda, todas ellas áreas sitas en el centro, sur y este de la Península y en el Valle del Ebro; excepcionalmente figura Gijón.

Los mínimos corresponden a áreas gallegas: Lugo, Orense, Pontevedra y Santiago; y.los inmediatos superiores a áreas del Valle del Duero (León, Zamora, Avila y Aranda de Duero) y a Santa Cruz de la Palma.

La moda (índices comprendidos entre 0,5001 y 0,6000 ) corresponde al grupo cuarto, y en él y en los grupos inmediatos se hallan situadas la mayor parte de las áreas, que, con pocas excepciones, pertenecen a todas las Regiones y ratifican la coexistencia de población rural o urbana en todas ellas y en el conjunto nacional. 